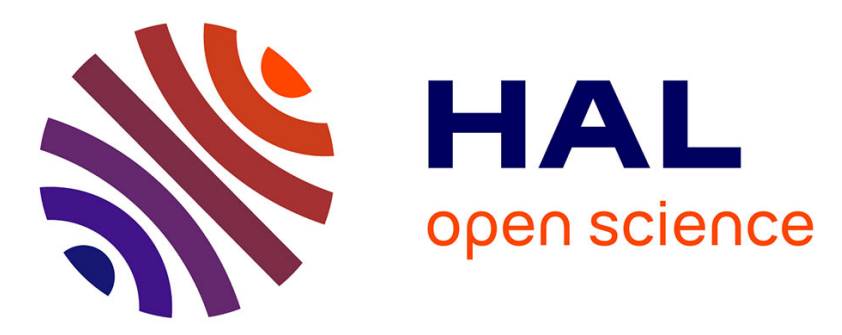

\title{
Imagerie cérébrale: vers une connaissance plus approfondie du système nerveux central
}

Giovanni de Marco, Geneviève Vens-Wagner, Monique Le Guen, Armande Le Pellec, Tarak Driss

\section{To cite this version:}

Giovanni de Marco, Geneviève Vens-Wagner, Monique Le Guen, Armande Le Pellec, Tarak Driss. Imagerie cérébrale: vers une connaissance plus approfondie du système nerveux central. Biologie Aujourd'hui, 2010, 204 (4), pp.321-331. 10.1051/jbio/2010027 . halshs-00573048

\section{HAL Id: halshs-00573048 \\ https://shs.hal.science/halshs-00573048}

Submitted on 10 Oct 2019

HAL is a multi-disciplinary open access archive for the deposit and dissemination of scientific research documents, whether they are published or not. The documents may come from teaching and research institutions in France or abroad, or from public or private research centers.
L'archive ouverte pluridisciplinaire HAL, est destinée au dépôt et à la diffusion de documents scientifiques de niveau recherche, publiés ou non, émanant des établissements d'enseignement et de recherche français ou étrangers, des laboratoires publics ou privés. 
Biologie Aujourd'hui, 204 (4), 321-331 (2010)

(C) Société de Biologie, 2011

DOI: $10.1051 /$ jbio/2010027

\title{
Imagerie cérébrale : vers une connaissance plus approfondie du système nerveux central
}

\author{
Giovanni de Marco, Geneviève Wagner, Monique le Guen, Armande Le Pellec et Tarak Driss \\ Université Paris Ouest - STAPS, 200 avenue de la République, 92001 Nanterre Cedex, France \\ Auteur correspondant: Giovanni de Marco, demarco.giovanni@gmail.com
}

Reçu le 18 mai 2010

\begin{abstract}
Résumé - L'imagerie fonctionnelle a permis de progresser de façon significative dans la connaissance du cerveau. Elle permet d'appréhender le fonctionnement cérébral tant dans des conditions physiologiques normales que pathologiques. Nous avons souhaité faire une mise au point sur les concepts et les approches d'analyse d'images qui permettent d'aborder l'interconnectivité cérébrale, en ayant pris soin de redéfinir au préalable et le plus rigoureusement possible les notions essentielles de la biologie du neurone qui constituent le socle des hypothèses et des modèles testés en neuroimagerie. L'étude de circuits spécifiques en réseaux va permettre de cerner de façon plus réaliste le fonctionnement dynamique du SNC qui sous-tend de nombreuses fonctions cérébrales. Deux méthodes de modélisation de l'activité et de l'interactivité cérébrales mesurées par IRMf sont présentées.
\end{abstract}

Mots clés : Imagerie / système nerveux central / connectivité cérébrale / modélisation / IRMf

\begin{abstract}
Brain imaging: towards a more detailed knowledge of the central nervous system.

Functional imaging allowed the knowledge of the brain to progress significantly. Recent findings about modeling of the activity and the interactivity measured by functional imaging are presented, they allow to apprehend brain functioning and brain plasticity both in normal and pathophysiological conditions. The study of specific circuits in networks should make it possible to define more realistically the dynamic functioning of the central nervous system which underlies numerous brain functions.
\end{abstract}

Key words: Imaging / CNS / brain connectivity / modelling / fMRI

\section{Le Darwinisme neuronal}

Le cerveau s'adapte en permanence à la variabilité de l'environnement. Cette capacité découle de son organisation anatomique et fonctionnelle, héritage adaptatif d'une longue histoire évolutive, laquelle, au gré des mutations, sélections, conservations, a produit un outil capable lors de son développement de s'auto-modeler sur l'environnement et le réel qu'il rencontre, puis de se ré-informer en permanence au fur et à mesure des expériences acquises, les « référentiels» de chacun étant des circuits préférentiels individuellement acquis. Ces expériences acquises colorent nos pensées, nos créations conceptuelles et notre conscience, lesquelles signent notre humanité. Elles ne peuvent se manifester sans le support fonctionnel que sont les réseaux neuronaux comme l'attestent les lésions cliniques et, ce que ne pouvaient imaginer Aristote et Descartes qui plaçaient la « raison $»$ par sa rigueur logique au-dessus des « passions » (émotions), censées nous éloigner de la vérité. Les pensées comme les émotions ne sont pas des entités sans support matériel. Notre cerveau gère tous les savoirs instinctifs et innés qui constituent notre mémoire 
d'espèce. Ces savoirs sont le résultat d'une accumulation biologiquement intégrée de connaissances adaptées aux conditions de vie qu'ont rencontrées nos générations d'ancêtres. Les savoirs progressivement assimilés par les structures neurales de notre espèce sont ceux qui étaient importants, au temps concerné, pour la survie ou la reproduction de ces ancêtres, selon la théorie de l'évolution de Darwin. Pour survivre, nous devrons établir 90 à $95 \%$ des connexions synaptiques efficientes de notre néo-cortex après la naissance car, pour être réellement fonctionnel, notre cerveau alors immature doit acquérir des données complémentaires non innées sur son environnement de vie.

\section{Le système neveux central}

Le système nerveux central (SNC) reçoit des informations en provenance du milieu extérieur et des informations issues du milieu intérieur (proprioception, intéroception). Les émotions et les états somatiques sont aussi des perceptions et doivent être à ce titre prises en compte par le SNC (Damasio, 1994). Les sous-systèmes responsables du contrôle de ces fonctions sont topographiquement bien repérés dans l'organisme. Toutes ces données sont traitées et intégrées par les centres nerveux : substance grise du cortex, de la moelle épinière et des noyaux gris dispersés dans la substance blanche. L'organisation hiérarchique s'est mise en place au cours de l'histoire évolutive des vertébrés, de telle sorte que puisse se réaliser l'intégration globale de toutes les données métaboliques et physiologiques nécessaires à la vie physiologique, psycho-sociale et intellectuelle. Gandolfo (2004) précise les six domaines où s'imbriquent et s'interconnectent un ensemble de fonctions régulatrices : (1) le cerveau est le centre régulateur du système de communication endocrine, via les molécules messagères hormonales transportées à distance par le réseau sanguin; (2) le cerveau est le centre modulateur de notre système immunitaire (reconnaissance du soi et du non-soi), maintien de notre intégrité interne biochimique et biologique, réactions défensives face aux bactéries et aux microbes par phagocytose et sécrétion d'anticorps spécifiques de l'agresseur (antigène); (3) le cerveau est le centre de régulation des rythmes biologiques; (4) le cerveau est l'organe de la vie affective et émotionnelle, comme l'apprentissage du langage nous l'a fait pressentir, les émotions étant une des modalités de la communication, i.e. l'information pour soi et en direction des autres; (5) le cerveau est le centre de la communication relationnelle : il régit nos relations sociales, via nos comportements, via les informations fournies par nos sens et notre capacité à percevoir les émotions, les nôtres et celles des autres; enfin, (6) le cerveau est le centre de la vie psychique mentale et spirituelle. L'activité cérébrale intéresse toutes les fonctions intellectuelles depuis la perception, la mémoire, la planification, la prise de décision, les commandes motrices, la conscience. Ce sont des aspects qui nous sont très familiers mais dont le soubassement biologique global reste ignoré de la plupart d'entre nous.

\section{Organisation anatomique et fonctionnelle du neurone et des synapses}

Le neurone est l'unité structurale et fonctionnelle du système nerveux central. Cette cellule est spécialisée dans le traitement de l'information.

Un neurone est constitué par un corps cellulaire (noyau et cytoplasme), des expansions, les dendrites et un unique axone. Les dendrites constituent les voies d'entrée des informations qui seront traitées par le corps cellulaire et sa machinerie biochimique. L'axone, long prolongement unique mais ramifié au niveau de l'arborisation terminale, véhicule le message global de sortie. La communication entre les neurones se fait par des jonctions discontinues appelées synapses. Il existe deux types de synapses : les synapses de nature électrique, peu nombreuses, et les synapses chimiques de loin les plus nombreuses. Pour ces dernières la transmission du message au niveau synaptique est finement modulée par la dynamique et «l'interactivité » de multiples acteurs biochimiques. On estime à un demi-milliard le nombre de synapses dans $1 \mathrm{~mm}^{3}$ de substance grise du cortex. On ne détaillera pas davantage si ce n'est pour s'attarder sur les phénomènes biochimiques multiples qui se produisent au niveau des synapses car c'est à ce niveau que se réalise l'intégration des signaux nerveux et que se réorganisent les « carrefours » des voies nerveuses et donc de l'information circulante. Comme nous le soulignerons par la suite, cette réorganisation structurelle est également fonctionnelle par modulation.

Il faut désormais noter le rôle des cellules de la névroglie, le tissu de soutien des neurones. Certaines cellules de la « glie » constituent le système immunitaire cérébral; d'autres cellules, les astrocytes, plus nombreux que les neurones, constituent une nouvelle entité fonctionnelle de régulation. Ces cellules nerveuses forment autour des synapses un manchon continu qui délimite un nouveau compartiment intérieur, dans lequel un environnement biochimique contrôlé module l'information nerveuse circulante. L'ensemble des neurones forme le « tissu nerveux », qui a la propriété d'être excitable et conducteur (Nelson, 2003).

Un consensus existe autour du nombre de 100 milliards de neurones. Un neurone peut être connecté de 


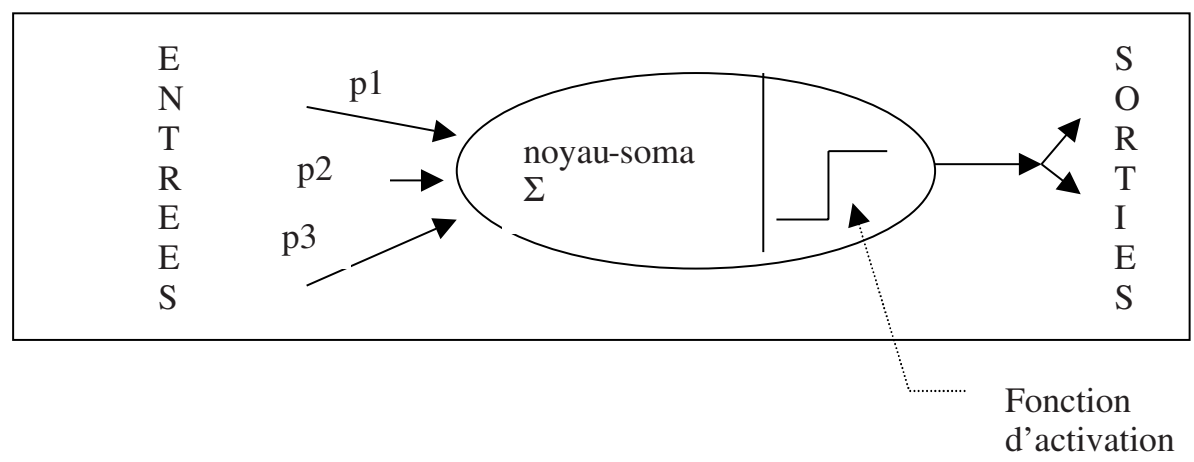

Fig. 1. Schéma d'un neurone artificiel montrant les entrées pondérées, la fonction de sommation et la fonction d'activation qui conduisent aux sorties.

1 à 10000 autres neurones, soit une combinatoire possible de $10^{15}$ connexions. Mis bout à bout, les câbles axoniques formeraient un circuit neuronal dont la longueur avoisinerait plusieurs centaines de kilomètres. On utilise souvent l'image d'autoroutes de l'information. La mise en place des 100 milliards de neurones au cours du développement s'effectue à un rythme de 4000 créations par seconde, rythme moyen qui, à certaines phases, dépasse 10000 créations par seconde (Jacquard, 1996). Le patrimoine génétique ne peut en définir que les grandes lignes; le contrôle rigoureux des structures réalisées ne serait pas possible. Ce sont les facteurs environnementaux et la sélection neuronale qui en modèleront l'organisation adaptée (épigenèse). Le message nerveux qui circule de neurone en neurone est, comme nous le verrons par la suite, une manifestation de type électrique mais il est généré en fait par des courants ioniques. Sa vitesse est de l'ordre de $1 \mathrm{~m}$ à $120 \mathrm{~m} / \mathrm{s}$ selon les fibres nerveuses. Cette vitesse s'explique par le fait que ce sont des migrations d'ions qui interviennent : le message nerveux est de nature électrochimique. Le passage contrôlé des ions se fait à travers des « canaux ioniques » spécifiques de chaque ion. Ces canaux sont des protéines, ils sont enchâssés dans la membrane du neurone. La structure et la (ou les) fonction (s) de ces protéines sont codées par les gènes.

Enfin, précisons que l'ensemble des cellules vivantes possède ce que l'on appelle un potentiel de repos qui correspond à l'existence d'une différence de potentiel entre l'extérieur et l'intérieur de la cellule. La différence de potentiel varie selon les types cellulaires entre $-40 \mathrm{mV}$ et $-150 \mathrm{mV}$, l'intérieur de la cellule est négatif par rapport au milieu extérieur du neurone qui, lui, est positif. Cette différence de potentiel est maintenue activement du vivant de la cellule. «Activement» signifie que cette activité est consommatrice d'énergie métabolique (ATP), cette activité disparaît donc avec la mort de la cellule. L'arrivée d'un signal (électrique ou biochimique), capable de modifier la perméabilité de la membrane à certains ions, pourra induire un nouveau message nerveux si les ions qui traversent cette membrane génèrent une dépolarisation suffisante. Ce message nerveux est appelé potentiel d'action. Si au contraire c'est une hyperpolarisation de la membrane qui se produit, il n'y aura pas de message nerveux généré, on dit qu'il y a inhibition (la synapse est inhibitrice).

\section{Le neurone : un microprocesseur biologique}

Du fait des modulations biochimiques du message nerveux au niveau de chaque synapse, le neurone post-synaptique se trouve soumis à une combinaison de signaux d'entrée issus des axones (et de leurs terminaisons) et des dendrites post-synaptiques qui leur sont connectées. Comme nous l'avons déjà évoqué, certaines connexions sont excitatrices, d'autres inhibitrices. Le neurone post-synaptique réalise l'intégration pondérée de l'ensemble des informations qu'il reçoit pour produire un nouveau potentiel d'action vers d'autres neurones post-synaptiques. Le neurone présynaptique produit « en sortie » un signal qui va activer en parallèle de nombreux autres neurones qui, à leur tour, constituent des systèmes de traitement (signaux en addition, en soustraction). Ce traitement en parallèle est une des raisons de l'efficacité incomparable du traitement de l'information chez l'Homme. C'est ce fonctionnement qui est mimé (sans la modulation fine et plastique permise par l'action biochimique) dans les réseaux de neurones artificiels et qui a amené les chercheurs en intelligence artificielle, et principalement les informaticiens statisticiens, à développer des algorithmes de calculs à base de réseaux de neurones (MacGregor, 1987). Chaque neurone est un automate très simple qui reçoit des impulsions de ses voisins (figure 1), et qui transmet lui-même une impulsion selon que la somme pondérée des entrées (avec un signe $>0$ 
pour une jonction excitatrice et un signe $<0$ pour une jonction inhibitrice) est supérieure à un certain seuil d'excitation (fonction d'activation). Les réseaux de neurones vont du réseau simple (deux neurones, un sensitif, un moteur pour un réflexe simple) aux réseaux les plus complexes (des milliers de neurones pour un seul réseau). La communication entre neurones peut revêtir une variété quasi illimitée de combinaisons spécifiques. Plusieurs centaines de messages différents peuvent parvenir, simultanément ou décalés dans le temps, sur un même neurone. Il existe donc une grande possibilité de modulation du message global de sortie. D'autant qu'au niveau de la synapse, différents types d'agents peuvent agir sur chacune des étapes biochimiques. On peut ainsi imaginer le gigantisme de la combinatoire possible. Il faut de plus y superposer un niveau supplémentaire de régulation assuré par les astrocytes de la névroglie. Selon Ascher (2002), une quarantaine de types de neurotransmetteurs ou médiateurs chimiques seraient connus dont les plus classiques sont l'acétylcholine, la noradrénaline, la dopamine (activateur central identifié dans différentes situations : motivation, interaction avec de nombreuses drogues, Parkinson), le glutamate (neurotransmetteur excitateur majeur associé à l'apprentissage et la mémoire), la sérotonine (dépression), les endorphines (connues des accros du sport). Néanmoins, avec les neuropeptides, on doit dépasser la centaine de médiateurs. Les synapses chimiques remplissent plusieurs rôles : des fonctions de valve (sens du message), d'amplificateur, de modulateur d'action et d'efficacité par les neurotransmetteurs. Ceux-ci peuvent provoquer soit une dépolarisation de la membrane, ils sont alors excitateurs (comme le glutamate), soit une hyperpolarisation, ils sont alors inhibiteurs de la transmission du message nerveux (comme le GABA). Il faut aussi signaler qu'après action, le neurotransmetteur libéré dans l'espace synaptique est recapturé par une protéine-transport pour être recyclé. De nombreuses drogues (cannabis, cocaïne, amphétamines, héroïne, et leurs dérivés) agissent au niveau de cette recapture. Si la recapture est inhibée, l'effet du neuromédiateur est prolongé; cet effet est souvent recherché. Cependant tous les neuromédiateurs ne sont pas inactivés par recapture.

L'activité corticale (frontale, pariétale, temporale, occipitale) et sous-corticale (limbique, thalamique, striatale, locus cœeruleus, noyau accumbens) serait le pendant d'une cascade d'événements métaboliques de nature électrique et biochimique qui se déroule au sein des cellules neuronales, gliales et en particulier des cellules astrocytaires situées à proximité des synapses (Heeger \& Ress, 2002). Ces cellules joueraient un rôle essentiel notamment dans la recapture du glutamate qu'elles délivrent aux terminaisons axonales sous la forme de glutamine. Cette activité synaptique et dendritique, intégrée sur quelques millimètres de tissu neural, présenterait un rendement énergétique en ATP relativement faible mais refléterait néanmoins les fluctuations lentes des potentiels de champ locaux, symbolisant des entrées électriques plutôt que des décharges de sortie. Les courants ioniques produits par le potentiel d'action des axones et les activités synaptiques génèrent des potentiels de champ extracellulaires (Logothetis et al., 2001). L'enregistrement de l'activité neuronale avec des microélectrodes, situées à distance d'un groupe de neurones, capte des signaux électriques, qui renseignent sur l'activité de sortie des neurones déchargeant ensemble de façon synchrone, et aussi sur l'activité synaptique et dendritique produite par les afférences (Hamalainen et al., 1993). Les deux informations peuvent être dissociées, l'une fournissant l'activité rapide de groupes de neurones et l'autre le potentiel lent de champ local (informations locales intégratives : potentiels post-synaptiques excitateur et inhibiteur, oscillation du potentiel de membrane).

\section{Imagerie et techniques de mesure de l'activité cérébrale}

L'essor de l'imagerie fonctionnelle, depuis une vingtaine d'années, a permis de progresser de façon significative dans la connaissance du cerveau, tout d'abord avec la tomographie par émission de positons (TEP) et ensuite avec l'imagerie par résonance magnétique fonctionnelle (IRMf). Ces deux techniques d'imagerie fonctionnelle sont sensibles à des variations locales de flux et de volume sanguins cérébraux. La caméra TEP permet d'étudier, de façon quantitative dans le cerveau, le devenir de l'oxygène-16 de l'eau remplacée par une molécule radiomarquée, l'oxygène-15, émettrice de positons. La présence de ces molécules d'eau isotopiques dans une région cérébrale est repérée par les émissions de positons détectées par les cristaux de scintillation de la caméra qui permettent de reconstruire les images tomographiques. L'IRMf présente l'avantage d'être sensible à de très faibles variations locales de la concentration sanguine en oxygène, d'être non invasive et d'avoir une excellente résolution spatiale comparée à la TEP.

Le cerveau génère des ondes électromagnétiques qui reflètent l'activité électrique des neurones. Aussi, des chercheurs recueillent, à partir de techniques électrophysiologiques comme l'électroencéphalographie (EEG), la magnétoencéphalographie (MEG), ou la stimulation magnétique transcrânienne (TMS) (Singh et al., 2002), des informations qui se déroulent à des échelles temporelles et spatiales très différentes, séparément ou à partir d'acquisitions simultanées TMS/EEG (Komssi \& Kahkonen, 2006), TMS/PET (Laird et al., 2008) ou EEG/IRMf (Goldman et al., 
2000). L'EEG et la MEG mesurent respectivement le potentiel électrique et le champ magnétique induits par les neurones. Les techniques EEG/MEG sont particulièrement sensibles à un très large spectre d'oscillations neuronales, aux variations de potentiels postsynaptiques, ainsi qu'aux décharges rapides pour des volumes de population neuronale relativement faibles (environ 50000 neurones). La TMS est une technique qui permet de stimuler de manière non invasive les couches superficielles du cortex, de manière à en modifier localement et réversiblement l'excitabilité neuronale. Cette technique rend possible l'étude des relations causales entre cerveau et cognition chez les sujets sains et permet de déterminer si une région cérébrale particulière est nécessaire à l'exécution normale d'un processus cognitif. La combinaison de ces différentes techniques devrait permettre de mieux comprendre les mécanismes à partir desquels l'apport vasculaire s'adapte aux changements de l'activité neuronale. Cette adaptation du couplage neurovasculaire serait restreinte localement dans l'espace et dans le temps. Le couplage des techniques électrophysiologiques et d'imagerie devrait permettre d'obtenir une représentation cérébrale de haute résolution spatio-temporelle, d'améliorer notre connaissance sur le couplage neuro-vasculaire et potentiellement de fournir des indications précoces sur les dysfonctionnements cérébraux (Lee et al., 2003). Seule l'IRMf sera détaillée dans la suite de cet article.

\section{Du neurone à l'activité vasculaire mesurée en IRMf (ou activité BOLD)}

$\mathrm{Au}$ cours d'une décharge neuronale, les variations hémodynamiques locales mesurées par IRMf sont faiblement corrélées avec l'activité rapide des neurones et fortement avec le potentiel lent de champ local. La mesure du signal en IRMf reflèterait ainsi l'activité d'entrée des neurones et les processus intracorticaux associés. Il a été montré également à partir d'enregistrements unitaires que la fréquence des décharges neuronales était globalement reliée au signal IRMf (Heeger \& Ress, 2002). Ainsi, le contraste obtenu en IRMf décrit également sous l'acronyme «BOLD » (Blood Oxygen Level Dependent) représenterait la signature des conséquences de l'activité électrique, énergétique et hémodynamique du cerveau. Malgré un certain nombre d'incertitudes sur la description exacte des mécanismes microscopiques à l'origine de l'activité neurovasculaire, une stimulation perceptive, cognitive ou somesthésique engendre dans différentes régions du cerveau une cascade de processus métaboliques qui accompagne l'activité neuronale. La réponse hémodynamique à cette demande implique une augmentation des flux et volume sanguins cérébraux qui varieront dans chaque voxel activé. Ce signal BOLD évoluera au cours du temps en fonction de la demande métabolique et de la localisation des régions cérébrales impliquées, en relation avec les conditions d'oxygénation, de flux et de volumes sanguins cérébraux.

L'IRMf utilise comme agent de contraste une substance endogène, l'hémoglobine contenue dans les hématies. Soumise à un champ magnétique statique, l'hémoglobine présente des propriétés magnétiques qui diffèrent suivant sa forme chimique oxydée ou réduite, i.e. suivant qu'elle est liée ou non à l'oxygène moléculaire. La désoxyhémoglobine (hémoglobine sans oxygène) présente des propriétés paramagnétiques (électrons non appariés) qui perturbent localement le champ magnétique (de Marco et al., 2006). Les différences de susceptibilité magnétique qui s'ensuivent, s'accompagnent d'une création de gradients de champ magnétique microscopiques intraglobulaires et intravasculaires qui vont s'étendre jusque dans le milieu périvasculaire (de Marco et al., 2004). La conséquence immédiate de ce champ inhomogène est un déphasage des spins (protons) tissulaires dans le voisinage des vaisseaux, une diminution du temps de relaxation transversale $\mathrm{T} 2 *$ et donc une chute de l'intensité de signal. Ainsi, le signal IRMf, induit et modulé par différents processus électriques et métaboliques, apparaît dans le voxel de l'image et dans les premiers instants de la mesure sous forme d'une intensité de signal légèrement diminuée (pas toujours observable sur les imageurs cliniques de $1,5 \mathrm{~T})$. On peut noter ici que cette réponse initiale, bien qu'inconstante, pourrait constituer un marqueur temporel précoce $(<1$ seconde) de l'activité neuronale et métabolique puisqu'elle précède les effets dynamiques de flux. L'accroissement de flux consécutif à l'activité métabolique va enrichir et diluer le compartiment vasculaire en oxyhémoglobine (hémoglobine chargée en oxygène) diamagnétique (appariement des électrons). Ce flux va avoir pour conséquence de réhomogénéiser le champ magnétique statique et donc d'induire une augmentation de l'intensité de signal qui atteindra un maximum (environ 4 à 6 secondes). L'amplitude maximale sera maintenue en fonction de la durée de la période d'activation. À l'arrêt de la phase d'activation, le flux et le volume sanguins vont diminuer, ceci se traduira par un retour progressif de l'intensité de signal vers la ligne de base.

Le contraste BOLD est obtenu classiquement en IRM à partir d'une séquence de type Echo-Planar (EPI) de type écho de gradient, sensibilisée aux effets de susceptibilité magnétique. Cette technique d'imagerie multicoupes représente à l'heure actuelle la technique d'acquisition la plus rapide et permet de couvrir l'ensemble du cerveau en moins de 2 secondes. Cette séquence présente une sensibilité accrue à de faibles 
variations locales du champ magnétique qui seront d'autant plus importantes que l'intensité du champ magnétique sera élevée. L'apport des hauts champs magnétiques (3T et au-delà) en IRMf est incontestable puisqu'ils permettent d'obtenir une meilleure résolution spatiale des images, avec la possibilité de diminuer la taille du voxel du fait d'une augmentation du rapport signal sur bruit, d'accroître la sensibilité au contraste BOLD (accroissement des effets de susceptibilité), d'améliorer la spécificité microvasculaire (de Marco et al., 2003) et de permettre une meilleure utilisation des paradigmes existants (e.g. événementiels) (Harel et al., 2006).

\section{De la biologie moléculaire aux réseaux neuronaux}

Notre cerveau est l'objet d'une trame programmée génétiquement (un tiers des gènes est affecté au cerveau). Au cours de la gestation, les neurones se développent en un quadrillage prolifique et aléatoire selon une séquence identique car spécifiée génétiquement mais déjà régulée par l'environnement interne local (embryogenèse et phénomènes d'induction). Au cours du développement postnatal, on constate : une réorganisation par élagage de certaines connexions, un renforcement ou un affaiblissement de certaines autres en réponse à des stimulations issues de l'environnement (épigenèse), voire des créations (épines dendritiques et dédoublement de synapses observables par imagerie cérébrale). Les nouvelles connexions créent des circuits privilégiés pour la circulation de l'information. Cette plasticité permet à nos circuits cérébraux, notamment ceux du néocortex, de s'organiser d'une façon qui correspond et s'adapte au monde dans lequel chaque individu vit et se développe. Notre patrimoine génétique commun est de 30000 gènes qui seront « traduits » en des milliers de protéines différentes. Le mécanisme de formation des synapses dépend de la maturation $\mathrm{du}$ système nerveux central, mais l'élimination et l'élagage dépendent de l'expérience. Les signaux issus de l'environnement agiront sur les gènes par l'intermédiaire d'interrupteurs qui sont des facteurs de transcription (Fields, 2005).

À la base de ce système centralisé, on trouve les fonctions primaires de survie appelées aussi fonctions végétatives qui sont chapeautées par trois systèmes généraux de contrôle d'information et de régulation des fonctions vitales : le système endocrinien, le système immunitaire qui permet le maintien de l'intégrité spécifique et individuelle, et d'autre part le système nerveux. Le système endocrinien contrôle les fonctions biologiques par voie chimique lente (minutes, heures, jours) via les hormones (messagers chi- miques véhiculés par voie sanguine). De nombreux autres « médiateurs chimiques » non hormonaux interviennent également dans la communication cellulaire; le système immunitaire contrôle l'intégrité du soi selon deux voies complémentaires et coopératives, une voie cellulaire (cellules immunitaires fixées ou circulantes) et une voie moléculaire. Enfin le système nerveux contrôle les fonctions de relation entre organes par voie rapide $(\mathrm{m} / \mathrm{s})$. Les voies « physiques » de communication sont représentées par les fibres des neurones. Les fibres périphériques regroupées en faisceaux forment les nerfs; les messages nerveux sont des signaux électriques codés en fréquence qui transitent au travers d'une vaste circuiterie.

En définissant des populations de neurones comme des réseaux locaux reliés transitoirement par des connexions dynamiques, réciproques et largement distribuées, Varela et al. (2001) proposent de faire une distinction entre des réseaux « locaux » et des réseaux « large échelle ». Un réseau local serait défini comme une quantité de tissu nerveux d'environ $1 \mathrm{~cm}^{3}$ qui synchroniserait son activité sur la base d'une cytoarchitecture locale et à partir de connexions monosynaptiques dont les délais de conduction atteindraient 4 à $6 \mathrm{~ms}$. Par exemple, dans les colonnes du cortex visuel primaire séparées de 2 à $7 \mathrm{~mm}$, les neurones partageant des propriétés similaires agiraient de façon synchrone. En revanche, les connexions dynamiques « large échelle » seraient définies comme des interactions entre des régions distantes reliées entre elles par des circuits de faisceaux de fibres. Un réseau large échelle concernerait des assemblées de neurones qui sont séparées de plus d'un centimètre et synchroniseraient leur activité à partir de voies polysynaptiques dont les délais de transmission dépasseraient les 8 à 10 ms. Par exemple, des assemblées de neurones situées entre les lobes occipital et frontal seraient séparées par des dizaines de millisecondes en temps de transmission. Cette dichotomie de la circuiterie du cerveau en réseaux « locaux » et « large échelle » respectivement servira de base neurale pour appuyer l'hypothèse selon laquelle l'architecture fonctionnelle cérébrale agira suivant les principes de ségrégation et d'intégration fonctionnelles (Hebb, 1949).

\section{Vers une modélisation de la circuiterie cérébrale par imagerie}

Le principe d'intégration fonctionnelle suppose qu'il existe une dynamique large échelle entre les régions interconnectées. Les fonctions cérébrales vont pouvoir s'exprimer au sein de circuits en réseau. Dans ce système, les fonctions cérébrales sont incluses dans des réseaux spécialisés qui se dynamisent en fonction de la demande (excitation/inhibition) et de la 
nature de la tâche cognitive (phénomènes d'adaptation, de répétition/suppression de l'activité). Les aires cérébrales recrutées sous-tendant plusieurs fonctions sont multisensorielles et peuvent successivement appartenir à des réseaux fonctionnels différents. Autrement dit, une aire cérébrale donnée ne présenterait pas une fonction unique, mais partagerait ses ressources qui seraient mises à profit dans de multiples circuits en réseau. Ce principe a été utilisé pour l'analyse des potentiels évoqués obtenus à partir d'enregistrements multipolaires (Gerstein \& Perkel, 1969). Bien que le principe de ségrégation se rapporte à des processus fonctionnels engagés spécifiquement dans des régions spécialisées et/ou localisées, nous savons maintenant que les fonctions du cerveau sont mieux appréhendées sur la base du principe d'intégration fonctionnelle. À partir d'une telle approche, ce ne sont pas seulement des régions cérébrales isolées qui sont supposées traiter l'information, mais un ensemble de régions cérébrales interconnectées qui interagissent de manière cohérente et dynamique. Ainsi sur la base du principe d'intégration, les relations fonctionnelles qui existent entre plusieurs régions cérébrales peuvent être analysées.

Le concept d'intégration fonctionnelle bien adapté à l'analyse des données en neuroimagerie va permettre d'identifier des systèmes fonctionnels distribués et d'étudier la connectivité fonctionnelle et effective. La connectivité fonctionnelle est définie comme une corrélation temporelle existant entre des évènements neurophysiologiques proches ou distants (Friston et al., 1993). La connectivité effective reflète l'influence qu'un système neural exerce sur un autre système neural (Friston, 1994). La connectivité effective dépend principalement d'un modèle théorique neurobiologique et/ou neuroanatomique faisant état de connaissances préalables d'aires interconnectées et d'un modèle mathématique spécifiant une structure du réseau à examiner. Les régions cérébrales activées et potentiellement connectées entre elles peuvent être mises en évidence en IRMf et à l'aide de différentes techniques multivariées d'analyse des données. Nous retiendrons les méthodes dites « data-driven » d'étude de la connectivité fonctionnelle, telles que l'analyse de la corrélation partielle (Rykhlevskaia et al., 2006 ; Marrelec et al., 2008), l'analyse en composantes principales (Friston et al., 1999), l'analyse en composantes indépendantes (McKeown et al., 1998), et l'analyse spectrale (Fall \& de Marco, 2007, 2008; Fall et al., 2007) que nous exposerons succinctement. Existent également les méthodes dites «hypothesis-driven » d'étude de la connectivité effective telles que la causalité de Granger (Roebroeck et al., 2005; Abler et al., 2006), les modèles autorégressifs (Harrison et al., 2003), les modèles structuraux (McIntosh \& Gonzalez-Lima, 1994; de Marco et al., 2009) et les modèles causaux dynamiques (Friston et al., 2003; Penny et al., 2004). La modélisation par équation structurelle (SEM) et la modélisation causale dynamique (DCM), que nous décrirons brièvement, permettent de représenter les régions fonctionnelles « activées » comme des circuits en interaction et de prendre en compte les flux dynamiques d'informations qui transitent au travers de ces différentes régions cérébrales (nœuds) spatialement distribuées (Varela et al., 2001).

L'analyse de la connectivité fonctionnelle dans le domaine spectral peut permettre de s'affranchir de la variabilité interrégionale de la fonction de réponse hémodynamique et de la nature multi-fréquentielle du signal BOLD. En effet, le signal mesuré en IRMf dans certaines régions du cerveau est souvent détérioré par un bruit physiologique de nature bien structurée, notamment celui des pulsations respiratoires et cardiaques. De telles variabilités interrégionales sont susceptibles d'affaiblir les corrélations estimées dans le domaine temporel et indépendamment de l'activité neuronale sous-jacente (Sun et al., 2004). Les artéfacts qui habituellement perturbent la forme de la fonction de réponse hémodynamique peuvent être plus facilement contrôlés dans le cas de l'utilisation d'une analyse spectrale du signal puisque les corrélations peuvent être calculées cette fois à une fréquence spécifique ou dans une bande de fréquences bien définie. La méthode spectrale permet d'extraire un ensemble de paramètres tels que la phase et la cohérence qui reflètent des informations précises sur la dynamique temporelle d'un signal supposé linéaire (Fall \& de Marco, 2008). L'analyse de cohérence appliquée à des signaux d'origine physiologique est pratiquée en EEG et en MEG. Elle a été appliquée aussi bien chez des sujets témoins (Zaveri et al., 1999) que dans le cas de patients présentant des crises d'épilepsie ou des tumeurs cérébrales (Halliday et al., 2000). En IRMf, l'analyse spectrale a permis d'identifier des patterns d'activités hémodynamiques dans le cas d'une tâche composée de stimuli visuels et auditifs (Marchini \& Ripley, 2000), de mettre en évidence dans les régions occipitales par exemple une dépendance entre la durée d'un stimulus visuel et le déplacement de la phase de la réponse hémodynamique (Muller et al., 2003), ou encore d'identifier des régions interconnectées lors d'une tâche visuo-motrice.

La SEM et le DCM représentent deux techniques de modélisation de l'interactivité cérébrale; elles supposent un modèle théorique et/ou empirique de connexions et l'existence de relations causales entre les régions impliquées dans le modèle. La SEM qui repose sur une matrice de variance/covariance des signaux enregistrés permet d'analyser la structure des données observées et confirme, suivant la complexité du modèle, les hypothèses théoriques. En bref, la SEM 


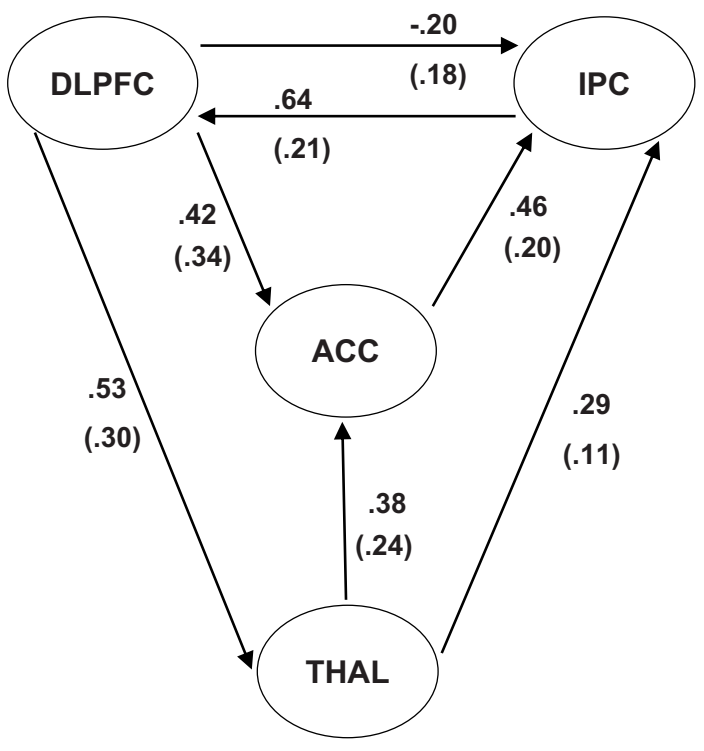

Fig. 2. Modèle SEM incluant le cortex préfrontal dorsolatéral (DLPFC), le cortex cingulaire antérieur (ACC), le cortex pariétal inférieur (IPC) et le thalamus (THAL). Les valeurs des coefficients de chemin obtenues respectivement dans la tâche d'alerte phasique et la tâche d'alerte intrinsèque (entre parenthèses) sont indiquées. Pendant la tâche d'alerte phasique, tous les coefficients de chemin sont statistiquement renforcés (d'après Périn et al., 2010).

représente une méthode statistique d'analyse multivariée des données qui consiste à confirmer un modèle théorique. Chaque relation (connexion) testée dans le modèle doit être fondée sur des bases théoriques. Ainsi la conception du cadre théorique nécessite une grande rigueur scientifique, la question expérimentale doit être clairement posée et pertinente, la problématique correctement argumentée et les hypothèses vérifiées. Cette méthode statistique considère que le cerveau fonctionne comme un système statique, que les neurones déchargent instantanément dans chaque région du cerveau et que l'activité se propage directement entre les différents nœuds fonctionnels du réseau supposé. Ainsi, les aires corticales recrutées au cours de l'activité et les fonctions cérébrales sont intégrées au sein de réseaux spécifiques. L'exemple de la figure 2 illustre un modèle SEM où les cercles représentent les nœuds du modèle et chaque flèche correspond à un circuit permettant de relier une région à une autre région du modèle. Dans cet exemple, les auteurs (Périn et al., 2010) ont étudié les mécanismes d'alerte en IRMf en utilisant une tâche de temps de réaction. Les valeurs (avec et sans parenthèses) représentent les coefficients de chemin des deux expériences qui ont été testées par le modèle (alerte intrinsèque et phasique respectivement). Dans cette étude, le modèle a confirmé le rôle prépondérant joué par le cortex préfrontal dorso- latéral dans le maintien de l'état d'alerte et dans le mécanisme de préparation temporelle engagée au cours de l'alerte phasique. Les lecteurs désireux d'approfondir cette technique de modélisation trouveront de nombreux détails méthodologiques dans l'article de de Marco et al. (2009).

L'approche de la connectivité effective peut être également abordée de manière déterministe à partir du formalisme des systèmes dynamiques ou DCM (Gössl et al., 2000; Friston et al., 2006). L'activité des réseaux neuronaux est reconstruite à partir de modèles neuronaux qui génèrent des signaux BOLD à partir d'un modèle vasculaire (Friston et al., 2000). La méthodologie est issue des neurosciences computationnelles (Hanson \& Olson, 1990) et s'appuie sur la résolution d'équations différentielles. Le cerveau est traité cette fois comme un système dynamique non linéaire (Friston et al., 2003). Le DCM s'appuie sur la prédiction des paramètres d'un modèle neuronal d'interaction biologiquement réaliste, fondé sur des hypothèses neurophysiologiques et neuroanatomiques propres à la tâche cognitive considérée. Les paramètres du modèle d'état d'entrée-sortie sont spécifiés et estimés sur la base : (1) de leur connectivité intrinsèque représentant les paramètres régulant le couplage entre les états du système; (2) de paramètres qui contrôlent l'influence d'entrées extrinsèques connues; (3) de paramètres bilinéaires utilisés pour moduler le couplage de la connectivité effective. L'identification et l'estimation des paramètres du modèle se font dans un cadre probabiliste (Friston, 2002). Cette approche de modélisation est appliquée pour analyser des circuits en réseau dont le flux d'informations dépend entièrement du contexte expérimental. Par exemple, à partir d'une activité synaptique donnée, les signaux BOLD associés peuvent être inférés. Bien que ce type de modélisation ne considère pas chaque neurone en particulier mais principalement des populations neuronales, nous devons faire face à une très grande complexité modélisatrice, notamment en termes de choix des paramètres du modèle. C'est pourquoi une approche probabiliste, en utilisant des informations $a$ priori dans l'optimisation des paramètres, est utilisée pour faire face aux indéterminations inhérentes à ce type d'approche. Une probabilité est interprétée comme la traduction numérique d'un état de connaissance (le degré de confiance accordé à une hypothèse). Cette méthode de modélisation suppose (comme de nombreuses approches de modélisation) des connaissances a priori sur le fonctionnement cérébral, comme l'efficacité synaptique, les populations de neurones recrutées au cours de la tâche, les mécanismes d'interaction mis en jeu ou la nature des connexions neuroanatomiques reliant les neurones entre eux. Ce dernier point est facilité par l'exploration de la connectivité anatomique ou neuroanatomie 


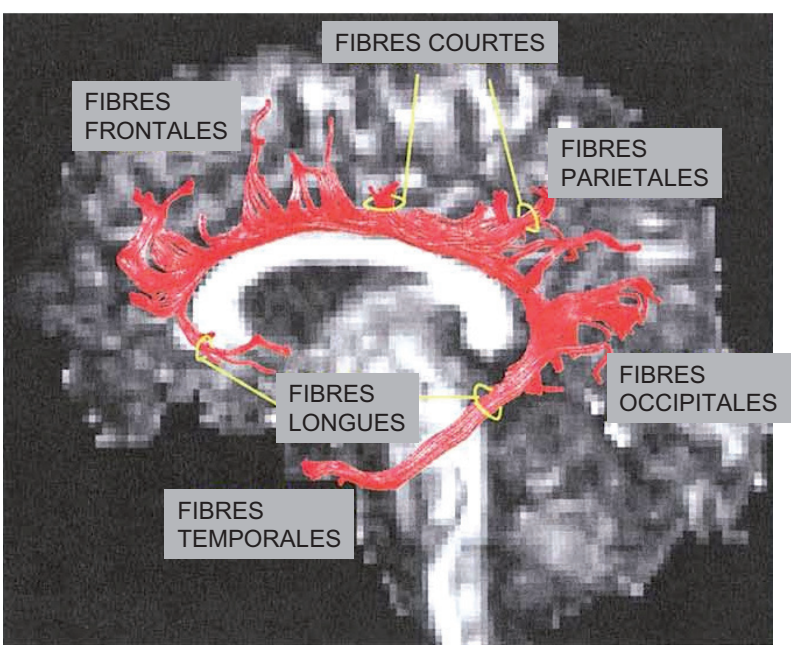

Fig. 3. Reconstruction tractographique par DTI d'une vue latérale du cingulum gauche. Les fibres longues connectent le lobe frontal au lobe temporal, alors que les fibres courtes relient les aires voisines du cingulum et du gyrus médian aux lobes frontal, pariétal, occipital et temporal (d'après Catani et al., 2002).

des faisceaux de fibres de substance blanche par imagerie en tenseur de diffusion (DTI). Les fibres de substance blanche cérébrale sont reconstruites à partir de la technique tractographique (Wakana et al., 2004). La figure 3 montre un exemple de fibres blanches cérébrales qui ont été reconstruites par DTI. Cette technique couplée à l'IRMf permet de mieux définir les connexions anatomiques directes ou indirectes reliant les différentes aires fonctionnelles de l'ensemble du cerveau (Shinoura et al., 2005). Elle permet de mieux cibler les circuits en réseau qui pourraient être potentiellement engagés dans différentes tâches.

\section{Conclusion}

Ainsi, l'analyse des images tractographiques associée à la connectivité cérébrale peut conduire à une meilleure visibilité des circuits en réseau recrutés au cours d'une tâche cognitive. De même, la combinaison de différentes techniques (EEG, EMG, IRMf, TMS) est prometteuse car elles apportent des renseignements distincts et complémentaires dans l'espace et dans le temps. Leur association devrait permettre à terme de mieux cerner les fonctionnements et les dysfonctionnements du SNC, également de mieux comprendre les mécanismes de couplage des activités électrique, métabolique et hémodynamique du cerveau. Les neuroscientifiques devraient pouvoir bénéficier dans les années à venir de l'évolution des différentes techniques électro-physiologiques et d'imagerie, des méthodes de modélisation de l'activité et de l'interactivité cérébrales qui permettront de mieux caractériser et quantifier les processus physiologiques et physiopathologiques du SNC.

\section{Nous n'avons pas de conflits d'intérêt.}

\section{Références}

Abler B., Roebroeck A., Goebel R., Hose A., SchonfeldtLecuona C., Hole G., Walter H., Investigating directed influences between activated brain areas in a motorresponse task using fMRI. Magn Reson Imaging, 2006, 24, 181-185.

Ascher P., Les signaux neuronaux, in Université de tous les savoirs, le Cerveau, le Langage, le Sens., O. Jacob, 2002, 59-73.

Catani M., Howard R.J., Pajevic S., Jones D.K., Virtual in vivo interactive dissection of white matter fasciculi in the human brain. NeuroImage, 2002, 17, 77-94.

Damasio A., Descartes' Error, Emotion, Reason and the Human Brain. Grosset/Putnam Books, 1994.

de Marco, G., Peretti I., Troprès I., Franconi J., HenryFeugeas M., Cerebral perfusion: Dynamic susceptibility contrast MR imaging. Part I: Principle and theory. Médecine Nucléaire - Imagerie fonctionnelle et métabolique, 2003, 27.

de Marco G., Dassonvalle P., Henry-Feugeas M.C., Onen F., Peretti I., Cerebral perfusion: dynamic suceptibility contrast MR imaging. Part 2: vascular models and data extraction. Médecine Nucléaire - Imagerie fonctionnelle et métabolique, 2004, 28, 35-48.

de Marco G., Roche A., Peretti I., Gossuin Y., Lehmann P., Menuel C., Vallée J.N., Muller R.N., MRI contrast agents: theoretical approch and relaxometric mechanisms. Médecine Nucléaire - Imagerie fonctionnelle et métabolique, 2006, 30, 645-658.

de Marco G., Vrignaud P., Destrieux C., de Marco D., Testelin S., Devauchelle B., Berquin P., Principle of structural equation modeling for exploring functional interactivity within a putative network of interconnected brain areas. Magn Reson Imaging, 2009, 27, 1-12.

Fall S., de Marco G., Functional exploration of the brain by fMRI. Neurophysiol Clin, 2007, 37, 229-237.

Fall S., de Marco G., Assessment of brain interactivity in the motor cortex from the concept of functional connectivity and spectral analysis of fMRI data. Biol Cybern, 2008, 98, 101-114.

Fall S., de Marco G., Assessment of brain interactivity in the motor cortex from the concept of functional connectivity and spectral analysis of fMRI data. Biological Cybernetics (in press).

Fall S., Lehmann P., Ambaiki K., Vallée J.N., Meyer M.E., de Marco G., Contribution of the spectral analysis to the brain connectivity study by fMRI. Neurophysiol Clin, 2007, 37, 239-247. 
Fields D., La force des souvenirs. Pour la Science, 2005, $\mathrm{n}^{\circ} 335$.

Friston K., Functional and effective connectivity in neuroimaging: a synthesis. Hum Brain Mapp, 1994, 2, 56-78.

Friston K., Bayesian estimation of dynamical systems: an application to fMRI. NeuroImage, 2002, 16, 513-530.

Friston K.J., Frith C.D., Liddle P.F., Frackowiak R.S., Functional connectivity: the principal-component analysis of large (PET) data sets. J Cereb Blood Flow Metab, 1993, 13, 5-14.

Friston K., Phillips J., Chawla D., Buchel C., Revealing interactions among brain systems with nonlinear PCA. Hum Brain Mapp, 1999, 8, 92-97.

Friston K., Mechelli A., Turner R., Price C., Nonlinear Responses in fMRI, The Ballon Model, Voltera Kernels, and Other Hemodynamics. NeuroImage, 2000, $12,466-477$.

Friston K.J., Harrison L., Penny W., Dynamic causal modelling. NeuroImage, 2003, 19, 1273-1302.

Friston K., Kilner J., Harrison L., A free energy principle for the brain. J Physiol, 2006, 100, 70-87.

Gandolfo G., À quoi sert le cerveau? Petite synthèse des grandes fonctions cérébrales. Revue de l'APBG, Biologie-Géologie, 2004, 3, 513-545.

Gerstein G.L., Perkel D.H., Simultaneously recorded trains of action potentials: analysis and functional interpretation. Science, 1969, 164, 828-830.

Goldman R.I., Stern J.M., Engel J., Jr., Cohen M.S., Acquiring simultaneous EEG and functional MRI. Clin Neurophysiol, 2000, 111, 1974-1980.

Gössl C., Auer D., Fahrmeir L., Dynamics models in fMRI. MRM, 2000, 43, 72-81.

Halliday D.M., Conway B.A., Farmer S.F., Shahani U., Russell A.J., Rosenberg J.R., Coherence between lowfrequency activation of the motor cortex and tremor in patients with essential tremor. Lancet, 2000, 355, 1149-1153.

Hamalainen M., Hari R., Ilmoniemi R., Knuutila J., Lounasmaa O., Magnetoencephalography - theory, instrumentation, and application to noninvasive studies of the working human brain. Rev Mod Physics, 1993, 65, 413-497.

Hanson S., Olson C., Connectionist Modeling and Brain Function: The Developing Interface. 1990, A Bradford Book., The MIT Press, Cambridge, Massachusetts.

Harel N., Ugurbil K., Uludag K., Yacoub E., Frontiers of brain mapping using MRI. J Magn Reson Imaging, 2006, 23, 945-957.

Harrison L., Penny W.D., Friston K., Multivariate autoregressive modeling of fMRI time series. NeuroImage, 2003, 19, 1477-1491.

Hebb D., The organization of Behavior: A Neurophysiological Theory. 1949, Wiley, New York.
Heeger D.J., Ress D., What does fMRI tell us about neuronal activity? Nat Rev Neurosci, 2002, 3, 142-151.

Jacquard A., La matière et la vie. Essentiels, Milan, 1996, $63 \mathrm{p}$.

Komssi S., Kahkonen S., The novelty value of the combined use of electroencephalography and transcranial magnetic stimulation for neuroscience research. Brain Res Rev, 2006, 52, 183-192.

Laird A.R., Robbins J.M., Li K., Price L.R., Cykowski M.D., Narayana S., Laird R.W., Franklin C., Fox P.T., Modeling motor connectivity using TMS/PET and structural equation modeling. NeuroImage, 2008, 41, 424-436.

Lee L., Harrison L.M., Mechelli A., A report of the functional connectivity workshop, Dusseldorf 2002. NeuroImage, 2003, 19, 457-465.

Logothetis N.K., Pauls J., Augath M., Trinath T., Oeltermann A., Neurophysiological investigation of the basis of the fMRI signal. Nature, 2001, 412, 150-157.

MacGregor R., Neural and Brain Modeling. 1987, Harcourt Brace Jovanovich, Publishers, New York, Academic Press, Inc.

Marchini L.J., Ripley B.D., A new statistical approach to detecting significant activation in functional MRI. NeuroImage, 2000, 12, 366-380.

Marrelec G., Kim J., Doyon J., Horwitz, B., Large-scale neural model validation of partial correlation analysis for effective connectivity investigation in functional MRI. Hum Brain Mapp, 2008, in press.

McIntosh A.R., Gonzalez-Lima F., Structural equation modeling and its application to network analysis in functional brain imaging. Hum Brain Mapp, 1994, 2, $2-22$.

Mckeown M.J., Makeig S., Brown G.G., Jung T.P., Kindermann S.S., Bell A.J., Sejnowski T.J., Analysis of fMRI data by blind separation into independent spatial components. Hum Brain Mapp, 1998, 6, 160-188.

Muller K., Mildner T., Lohmann G., von Cramon D.Y., Investigating the stimulus-dependent temporal dynamics of the BOLD signal using spectral methods. J Magn Reson Imaging, 2003, 17, 375-382.

Nelson P.G., Un tissu de plus en plus excitable. Science et Vie, 2003, 210, 81-86.

Penny W.D., Stephan K.E., Mechelli A., Friston K.J., Modelling functional integration: a comparison of structural equation and dynamic causal models. NeuroImage, 2004, 23, S264-S274.

Périn B., Godefroy O., Fall S., de Marco G., Alertness in young healthy subjects: an fMRI study of brain region interactivity enhanced by a warning signal. Brain Cogn, 72, 271-281.

Roebroeck A., Formisano E., Goebel R., Mapping directed influence over the brain using Granger causality and fMRI. NeuroImage, 2005, 25, 230-242. 
Rykhlevskaia E., Fabiani M., Gratton G., Lagged covariance structure models for studying functional connectivity in the brain. NeuroImage, 2006, 30, 1203-1218.

Shinoura N., Suzuki Y., Yamada R., Kodama T., Takahashi M., Yagi K., Fibers connecting the primary motor and sensory areas play a role in grasp stability of the hand. NeuroImage, 2005, 25, 936-941.

Singh K.D., Barnes G.R., Hillebrand A., Forde E.M., Williams A.L., Task-related changes in cortical synchronization are spatially coincident with the hemodynamic response. NeuroImage, 2002, 16, 103-114.

Sun F.T., Miller L.M., D'Esposito M., Measuring interregional functional connectivity using coherence and partial coherence analyses of fMRI data. NeuroImage, 2004, 21, 647-658.

Varela F., Lachaux J.P., Rodriguez E., Martinerie J., The brainweb: phase synchronization and large-scale integration. Nat Rev Neurosci, 2001, 2, 229-239.

Wakana S., Jiang H., Nagae-Poetscher L.M., van Zijl P.C., Mori S., Fiber tract-based atlas of human white matter anatomy. Radiology, 2004, 230, 77-87.

Zaveri H.P., Williams W.J., Sackellares J.C., Beydoun A., Duckrow R.B., Spencer S.S., Measuring the coherence of intracranial electroencephalograms. Clin Neurophysiol, 1999, 110, 1717-1725. 